\title{
Hydrophobic nanocrystals coated with an amphiphilic polymer shell: a general route to water soluble nanocrystals
}

\author{
Teresa Pellegrino ${ }^{1,2}$, Liberato Manna ${ }^{1,3, *}$, Stefan Kudera ${ }^{1}$, Tim Liedl ${ }^{1}$, \\ Dmitry Koktysh ${ }^{1}$, Andrey L. Rogach ${ }^{1}$, Simon Keller $^{1}$, Joachim \\ Rädler ${ }^{1}$, Giovanni Natile ${ }^{2}$ and Wolfgang J. Parak ${ }^{1, *}$ \\ ${ }^{1}$ Department of Physics \& Center for Nanoscience, Ludwig- \\ Maximilians Universität München, München, Germany \\ ${ }^{2}$ Department of Chemistry and Pharmacology, University of Bari, \\ Bari, Italy \\ ${ }^{3}$ National Nanotechnology Lab of INFM, Via Arnesano, Lecce, Italy
}

\section{Supporting information}

\section{Materials and Methods}

All chemicals were purchased from the various vendors, they were degassed (when not shipped under inert gas by the vendor), stored in the glove box and used without any further purification. All solvents used for the synthesis and the purification of nanocrystals (except for gold) and prior to polymer coating where anhydrous. All syntheses and purifications of nanocrystals (except for gold) and prior to polymer coating were carried out under argon. 


\section{Nanocrystal syntheses}

Colloidal nanocrystals of different materials were synthesized according to standard procedures

described in the literature $\left(\mathrm{CoPt}_{3}{ }^{1}, \mathrm{Au}^{2}, \mathrm{CdSe} / \mathrm{ZnS}{ }^{3}\right.$, and $\left.\mathrm{Fe}_{2} \mathrm{O}_{3}{ }^{4}\right)$ with few changes.

\section{Synthesis of colloidal CdSe/ZnS nanocrystals}

CdSe nanocrystals of different sizes were synthesized according to the procedure described by Reiss and coworkers ${ }^{5}$, with minor changes. In a typical synthesis, $5.75 \mathrm{~g}$ of hexadecylamine (HDA, technical grade, Sigma-Aldrich \#H7.40-8), $2.26 \mathrm{~g}$ of trioctylphosphine oxide (TOPO, 99\%, Sigma-Aldrich \#22.330-1), 2.20 g of dodecylphosphonic acid (DDPA 98\%, Polycarbon Inc.) and $501 \mathrm{mg}$ of CdO (99.99+\%, Sigma-Aldrich \#20.289-4) were mixed in a $50 \mathrm{ml} 3$-necked flask connected to an argon-vacuum line assembled in an argon glove-box. The mixture was degassed at $120{ }^{\circ} \mathrm{C}$ for 20 minutes and then heated to $300-320{ }^{\circ} \mathrm{C}$ until it turned clear and colorless. $1 \mathrm{ml}$ of tributylphosphine (TBP, 99\%, Strem \#15-5800) was then injected in the flask and the temperature was lowered to $270{ }^{\circ} \mathrm{C}$. In a vial, $320 \mathrm{mg}$ of Se powder $(99.99 \%$, SigmaAldrich \#22.986-5) were dissolved in $1.28 \mathrm{~g}$ of TBP under vigorous stirring and the resulting solution was injected in the flask. After injection, the temperature dropped by roughly $20{ }^{\circ} \mathrm{C}$ but was allowed to recover to $270{ }^{\circ} \mathrm{C}$ and was then maintained at this level throughout the synthesis. 3-5 minutes after the injection the color of the solution turned from colorless to yellow-orange, indicating the nucleation of CdSe nanocrystals. This color gradually turned to red, dark red and finally to brown 25-30 minutes after the injection (first exciton absorption peak at 620-630 nm). The reaction was monitored by taking aliquots during the growth, by diluting them in chloroform and by recording their UV-visible absorption spectra. The growth rate slightly varied from synthesis to synthesis, but it was always sufficiently low so that the synthesis could be stopped whenever the first exciton peak in the absorption spectrum reached a desired value, which could be easily correlated to the nanocrystal average size through calibration curves available from the literature ${ }^{6}$. The synthesis was stopped by removing the heating mantle and by rapidly cooling the flask. When nanocrystals with remarkably small average diameter were required ( $2 \mathrm{~nm}$ or less), the synthesis could be stopped few minutes after the injection, by quickly injecting 3-4 ml of toluene. However, the size distribution of these samples was always quite broad, and a size selective precipitation was required. The smallest, size-selected nanocrystal samples synthesized by this method had their first exciton absorption peak at around $480 \mathrm{~nm}$.

After stopping the reaction, the flask was cooled to $100{ }^{\circ} \mathrm{C}$ and $20 \mathrm{ml}$ of nonanoic acid (97\%, Sigma-Aldrich \#N5502) were added. The solution was stirred for additional 10-15 minutes at this temperature and then cooled to room temperature. The addition of an alkyl acid (carboxylic or phosphonic) avoided the formation of a gel when the original growth solution was cooled below $50{ }^{\circ} \mathrm{C}$. This gel did not dissolve in any of the tested solvents (chloroform, toluene, methanol, hexane, tetrahydrofuran) at room temperature and practically made the nanocrystal-surfactant mixture intractable. Addition of 5-10 $\mathrm{ml}$ of methanol to the mixture of nonanoic acid and growth solution caused the flocculation of nanocrystals, which could be separated by centrifugation. The precipitate was then dissolved in $10 \mathrm{ml}$ of toluene and the mixture was centrifuged for 30 minutes at $3000 \mathrm{rpm}$ in a $20 \mathrm{ml}$ vial to remove any residual gel present, which was collected at the bottom of the vial. The precipitate was discarded, and the supernatant was mixed with methanol to precipitate the nanocrystals. This precipitate was then redissolved in toluene, methanol was again added to it and the solution was centrifuged again. This step was repeated once more, after which the precipitate was dried under vacuum and stored as a powder in the dry box. This synthesis 
usually yielded between 200 and $400 \mathrm{mg}$ of nanocrystals in total, depending of the average nanocrystal size.

For the $\mathrm{ZnS}$ shell growth, $12 \mathrm{~g}$ of TOPO (technical grade, Alfa Aesar \#14114) were weighted in a $50 \mathrm{ml} 3$-necked flask, pumped to vacuum at $120{ }^{\circ} \mathrm{C}$ for 20 minutes. One $\mathrm{ml}$ of Trioctylphosphine (technical grade, Sigma-Aldrich \#11.785-4) was injected into the flask. $60 \mathrm{mg}$ of a dry nanocrystal powder were dissolved in $3 \mathrm{ml}$ of chloroform and the resulting solution was injected into the flask. The chloroform was removed by evacuating the flask for few minutes. The temperature of the mixture was then raised to $160^{\circ} \mathrm{C}$. The stock solution for the $\mathrm{ZnS}$ growth was freshly prepared by dissolving $0.647 \mathrm{~g}$ of a solution of diethylzinc $\left(\mathrm{C}_{4} \mathrm{H}_{10} \mathrm{Zn}, 1.0 \mathrm{M}\right.$ solution in heptane, Sigma-Aldrich \#40.602-3) and $0.19 \mathrm{~g}$ of hexamethyldisilathiane $\left(\mathrm{C}_{6} \mathrm{H}_{18} \mathrm{Si}_{2} \mathrm{~S}\right.$ ) (Aldrich \#28.313-4) in $4.73 \mathrm{~g}$ of TBP. Several drop-wise injections of this solution were done (1 ml each) at $160{ }^{\circ} \mathrm{C}$. The waiting time between injections was of the order of 5-10 minutes, during which it was assumed that the precursors injected had completely reacted. The injections were done dropwise, to allow a slow and uniform shell growth and to prevent the nucleation of $\mathrm{ZnS}$ crystals. After each injection, a small aliquot (10-20 $\mu$ l) of solution was extracted via a syringe, dissolved in $2 \mathrm{ml}$ of chloroform and its optical absorption and emission spectra were recorded. The optical densities of these aliquots were adjusted to 0.2 at the excitation wavelength, usually chosen in the 450-500 nm range. After the first few injections, the luminescence from the nanocrystals increased, indicating the growth of a $\mathrm{ZnS}$ shell. The luminescence then reached a maximum intensity, which was dependent on the particular nanocrystal size, and then it decreased upon additional injections. This decrease indicated that the shell grew over its optimal thickness and that it started developing defects. The optimal number of injections which maximized the final fluorescence quantum yield varied by synthesis, and ranged from 3-4 for the largest nanocrystal sizes to 6-7 for the smallest nanocrystals. After the injections were completed, the solution was cooled to $60{ }^{\circ} \mathrm{C}$ and $3 \mathrm{ml}$ of butanol were added in order to avoid solidification and to quench the residual unreacted chemicals. The solution was then transferred into a vial and stored for the polymer coating experiments.

\section{Synthesis of colloidal Au nanocrystals}

Colloidal Au nanocrystals were synthesized according to standard protocols ${ }^{2,7-9}$. All glassware was carefully cleaned in a $\mathrm{KOH} /$ isopropanol bath and carefully rinsed with water before use. All reactions were carried out at room temperature under ambient conditions. Briefly, $2.17 \mathrm{~g}$ of tetraoctylammonium bromide (TOABr, 98\%, Sigma-Aldrich \#29.413-6) were dissolved in $80 \mathrm{ml}$ of HPLC grade toluene and $300 \mathrm{mg}$ hydrogen tetrachloroaurate (III) (99.9\%, Alfa Aesar \#12325) were dissolved in $25 \mathrm{ml}$ Millipore water to yield a yellow translucent solution. The two solutions were mixed in a $100 \mathrm{ml}$ separation funnel and the funnel was shaken for about 5 minutes. During this time the initially colorless toluene phase (on the top) turned red and the initially yellow aqueous phase (on the bottom) turned colorless, indicating the formation of tetraoctylammoniumgold ion pairs. The aqueous phase was discarded and the toluene phase was transferred to a 100 $\mathrm{ml}$ round flask. In a beaker, $334 \mathrm{mg}$ of sodium borohydride (98\%, Sigma-Aldrich \#45.288-2) were dissolved in $25 \mathrm{ml}$ of Millipore water under vigorous stirring by means of a glass rod. This clear solution was then pipetted dropwise within one minute into the red solution of tetraoctylammonium-gold in toluene. Upon stirring for few seconds, the initial two phases started to mix and the color changed from red to red-violet. This color change indicates the nucleation of gold clusters mediated by sodium borohydride. The residual sodium borohydride in solution 
reduces the remaining gold ions, providing additional monomers for the growth of the nuclei. The solution was stirred for 1 hour, then it was transferred to a clean separation funnel and $25 \mathrm{ml}$ of $0.01 \mathrm{M} \mathrm{HCl}$ were added in order to remove the excess sodium borohydride. The funnel was shaken for 1 minute and the aqueous phase (on the bottom) was discarded. $25 \mathrm{ml}$ of $0.01 \mathrm{M}$ $\mathrm{NaOH}$ were added to the funnel to remove the excess acid and after shaking for 1 minute the aqueous phase was again discarded. Finally, $25 \mathrm{ml}$ of Millipore water were added to remove excess ions, the funnel was shaken for 1 minute and the aqueous phase was discarded. This last washing step was repeated 4 more times. The final solution was then transferred to a $100 \mathrm{ml}$ round flask and stirred for 1 day to allow the particles to Ostwald ripen to a thermodynamically stable average size and size distribution.

After the synthesis, a surfactant exchange procedure was carried out. Ten ml of 1-dodecanethiol (98\%, Sigma-Aldrich \#47,136-4) were added to the Au nanocrystals dissolved in toluene. The solution was heated to $65{ }^{\circ} \mathrm{C}$ and stirred for 2 hours. During this process the mercapto groups of the dodecanethiol molecules displace the $\mathrm{Br}^{-}$ions and yield dodecanethiol coated $\mathrm{Au}$ nanocrystals. The solution was then cooled to room temperature and the Au nanocrystals were precipitated by the addition of about $30 \mathrm{ml}$ of methanol (methanol was added until the solution turned cloudy), followed by centrifugation. After discarding the supernatant, the precipitate was dissolved in $10 \mathrm{ml}$ of toluene upon vigorous shaking, and the nanocrystals were precipitated again by the addition of about $4 \mathrm{ml}$ of cold $\left(-20^{\circ} \mathrm{C}\right)$ methanol (methanol was added until the solution turned cloudy) followed by centrifugation. The supernatant was discarded and the precipitate was redissolved in $2 \mathrm{ml}$ of toluene. In order to improve the size distribution of the sample, a size selective precipitation was carried out. Addition of $20 \mu \mathrm{l}$ of cold $\left(-20^{\circ} \mathrm{C}\right)$ methanol, followed by centrifugation, caused the precipitation of only the largest nanocrystals from the solution. They were discarded and the supernatant was used as final solution. The average diameter of the $\mathrm{Au}$ nanocrystals was estimated by TEM (hence not considering the surfactant shell) and the particle concentration was determined by measuring the optical absorption of the nanocrystal solution. For Au nanocrystals with a core diameter of $4.0 \mathrm{~nm}$ we assumed an optical extinction coefficient of $8.7 \cdot 10^{6} \mathrm{M}^{-1} \mathrm{~cm}^{-1}$ at their plasmon peak around $518 \mathrm{~nm}$. The concentration of the resulting Au nanocrystal solution was typically in the $\mu \mathrm{M}$ range.

\section{Synthesis of colloidal $\mathrm{CoPt}_{3}$ nanocrystals}

$\mathrm{CoPt}_{3}$ nanocrystals were synthesized according to the procedure described by Shevchenko and coworkers ${ }^{1}$. $0.033 \mathrm{~g}$ of Platinum (II) acetylacetonate (98\%, Strem \#78-1400), $0.25 \mathrm{~g}$ of 1adamantanecarboxylic acid (99+\%, Sigma-Aldrich \#01823) and $0.13 \mathrm{~g}$ of 1,2-hexadecanediol (technical grade, Sigma-Aldrich \#21.374-8) were dissolved in a mixture of hexadecylamine (4.0 g, technical grade, Sigma-Aldrich \#H7.40-8) and diphenyl ether (2.0 ml, 99+\%, Sigma-Aldrich \#24.083-4) and heated to $65{ }^{\circ} \mathrm{C}$ in a $50 \mathrm{ml}$ three-necked flask. At this temperature the surfactants melted and the resulting clear, yellowish solution was degassed for several minutes. The stock solution for the cobalt precursor was prepared by mixing $0.015 \mathrm{~g}$ of $\mathrm{Co}_{2}(\mathrm{CO})_{8}$ (Strem \#27-0400) with $0.7 \mathrm{ml}$ of 1,2-dichlorbenzene (99\%, Sigma-Aldrich \#24.066-4) in a small vial. The reaction mixture was heated to $200{ }^{\circ} \mathrm{C}$, and the stock solution for the cobalt precursor was injected into the flask through a syringe, under vigorous stirring. The solution was kept at $200{ }^{\circ} \mathrm{C}$ for $30 \mathrm{~min}$, then at $290{ }^{\circ} \mathrm{C}$ for $30 \mathrm{~min}$, and it was finally cooled to room temperature. Under these conditions relatively monodisperse $\mathrm{CoPt}_{3}$ nanocrystals with an average diameter of $8.0 \mathrm{~nm}$ are formed. If 
smaller particles are desired, a larger amount of $\mathrm{Co}_{2}(\mathrm{CO})_{8}$ should be dissolved in the precursor stock solution and the injection should be performed at a lower temperature ${ }^{10}$.

\section{Synthesis of colloidal $\mathrm{Fe}_{2} \mathrm{O}_{3}$ nanocrystals}

$\gamma-\mathrm{Fe}_{2} \mathrm{O}_{3}$ nanocrystals were prepared according to the method described by Hyeon and coworkers ${ }^{4}$. Briefly, $10 \mathrm{ml}$ of octyl ether (99\%, Sigma-Aldrich \#24.959-9) and $1.28 \mathrm{~g}$ of oleic acid (99+\%, Sigma-Aldrich \#75.090) where degassed in a $50 \mathrm{ml}$ flask at $60{ }^{\circ} \mathrm{C}$ for 20 minutes. $0.29 \mathrm{ml}$ of $\mathrm{Fe}(\mathrm{CO})_{5}\left(99.999 \%\right.$, Sigma-Aldrich \#48.171-8) were then added at $100{ }^{\circ} \mathrm{C}$ and the solution was heated up to refluxing temperature $\left(\sim 295-300{ }^{\circ} \mathrm{C}\right)$ for 1 hour. In case of insufficient cooling of the refluxing funnel oleic acid evaporated and the solution turned cloudy, which could be reverted by adding a small amount of oleic acid. This was especially problematic if the amount of iron precursor was too low. During the temperature rise the initial yellow-orange solution turned to pale yellow and sometimes to almost colorless. This color change was somehow different from what reported by Hyeon and coworkers and it initially induced us to believe that the synthesis was heading to failure. However, the solution almost suddenly turned to black during the refluxing, which was allowed to continue for the planned time (1 hour). The flask was then cooled down to room temperature, $0.34 \mathrm{~g}$ of $\left(\mathrm{CH}_{3}\right)_{3} \mathrm{NO}(98 \%$, Sigma-Aldrich \#31.759-4) were added and the mixture was heated at $130{ }^{\circ} \mathrm{C}$ for two hours, during which time it turned to dark brown. The flask was heated again to refluxing temperature for 1 hour, after which the solution was cooled to room temperature. Methanol was added to precipitate the nanocrystals, which were recovered upon centrifugation and redissolution in toluene. This synthesis usually yielded nanocrystals with a residual distribution of sizes. The largest nanocrystals, scarcely soluble in toluene, were removed simply by centrifugation of the toluene solution at $3000 \mathrm{rpm}$ for 30 minutes. The smallest nanocrystals were removed by size selective precipitation, by discarding the supernatant and by redissolving the precipitate in toluene. This size selective precipitation was quite easy since small nanocrystals hardly precipitated even upon the addition of several $\mathrm{ml}$ of methanol. The final, size-selected nanocrystals were redissolved in toluene and re-precipitated with methanol two additional times. This procedure helped to remove the excess surfactant and the residual $\left(\mathrm{CH}_{3}\right)_{3} \mathrm{NO}$. Removal of $\left(\mathrm{CH}_{3}\right)_{3} \mathrm{NO}$ had to carried out carefully, since the addition of large amounts of methanol to precipitate the nanocrystals from the toluene solution also caused the precipitation of the amine $\mathrm{N}$-oxide. 


\section{General description of the polymer coating procedure}

Before undergoing the polymer coating procedure, the nanocrystals were cleaned by removing the excess unbound surfactant. This was done by precipitating the nanocrystals with the addition of an appropriate solvent, discarding the supernatant and redissolving the precipitate in chloroform. This precipitation/redissolution step was done twice for $\mathrm{Au}$ and three times for $\mathrm{CoPt}_{3}$ and $\mathrm{Fe}_{2} \mathrm{O}_{3}$. For $\mathrm{CdSe} / \mathrm{ZnS}$ and $\mathrm{Au}$ an additional round of precipitation/redissolution usually resulted in a significant loss of solubility of the nanocrystals in chloroform.

For each sample, the average diameter of the inorganic nanocrystal cores was determined by TEM analysis. The overall surface area of each nanocrystal was calculated by considering the effective nanocrystal diameter $\left(\mathrm{d}_{\mathrm{eff}}(\mathrm{nm})=\mathrm{d}+2\right)$ as the sum of the diameter of its inorganic core (d) plus the surfactant shell, which was assumed to be $1 \mathrm{~nm}$ in thickness. The concentration of the starting nanocrystal solution was determined either by gravimetric methods or by absorption measurements when extinction coefficients were available from the literature. In order to avoid cross-linking effects among nanocrystals, the concentration of the initial nanocrystal solution was always adjusted to $0.5-2 \mu \mathrm{M}$ by dilution with chloroform.

A solution of Poly(maleic anhydride alt-1-tetradecene) (Sigma-Aldrich \#45.251-3) in chloroform (400mg/10ml, $0.136 \mathrm{M})$, henceforth referred to as "polymer solution", a solution of Bis(6aminohexyl)amine (Fluka \#14506) in chloroform (43 mg/10 ml, $0.02 \mathrm{M}$ ), henceforth referred to as "cross-liker solution", and a 0.5 x Tris-Borate-EDTA (TBE, Sigma-Aldrich \#T-3913) buffer solution, henceforth referred to as "buffer solution", were prepared and stored as stock solutions for all the polymer coating experiments. In poly(maleic anhydride alt-1-tetradecene) each polymer chain had an average length of 30 polymer units

The polymer solution was added to the nanocrystal solution. After stirring the resulting solution for 2 hours at room temperature, the chloroform solvent was slowly evaporated under reduced pressure by using a rotavapor system (Laborota 400, Heidolph). The cross-linker solution was then added to crosslink the polymer shell that had formed around each nanocrystal. The solution was then sonicated for 20 minutes, after which the solvent was slowly evaporated. The resulting powder was dissolved in the buffer solution to yield again a nanocrystal concentration in the range between 0.5 and $2 \mu \mathrm{M}$. After sonication for five minutes, the polymer-coated nanocrystals dissolved completely in the buffer solution. A filtration through a syringe membrane filter (Roth \#P818.1, $0.22 \mu \mathrm{m}$ pore size) helped to remove most of the excess of unbound polymer. The buffer was then exchanged with water by 2 rounds of dilution with water and re-concentration through a 100000 MWCO Centricon centrifuge filter (Millipore \#4424). Periodically, the centrifugation was paused and the sample was mixed to accelerate the passage of water through the filter. Finally, the remaining unbound polymer was removed by 2 consecutive purifications steps on a size exclusion column (sephadex superfine G200, Sigma-Aldrich \#84.961). Optionally, particles could be further purified by gel electrophoresis ${ }^{11}$.

Several polymer coating experiments have been performed. In these experiments the parameters that were varied are the number of polymer units per $\mathrm{nm}^{2}$ of nanoparticle surface and the ratio of polymer chains per cross-linker molecules (table 1). All experiments led to a successful transfer of the nanocrystals in water. We then decided to adopt a general protocol for the coating the nanocrystals, regardless of nanocrystal material. In this protocol the volumes of polymer and cross-linker solutions mixed with the nanocrystal solution are such that 100 polymer units are 
added per $\mathrm{nm}^{2}$ surface area of nanoparticles and the ratio of polymer chains to cross-linker is equal to 10. The application of this protocol is reported in detail for $\mathrm{Fe}_{2} \mathrm{O}_{3}, \mathrm{Au}, \mathrm{CdSe} / \mathrm{ZnS}$, and $\mathrm{CoPt}_{3}$ nanocrystals. Although the protocol worked satisfactorily for all the nanocrystal samples tested, it is likely that for each nanocrystal material and surfactant coating the parameters can be finely tuned to optimize the finale sample quality.

In addition, control experiments were performed to confirm that indeed the combined effect of the polymer and of the cross-linker was responsible for the water solubility of the nanocrystals. Nanocrystals to which only the polymer solution was added, but which did not undergo the crosslinking step, did not dissolve in water. Likewise, nanocrystals to which only the cross-linker solution was added did not dissolve in water.

The choice of cross-linker molecule is not unique and we are confident that in principle a molecule carrying at least two amino groups, sufficiently separated from each other, can be a suitable candidate. When 1,4-diaminobutane was used for instance as cross-linker instead of bis(6-aminohexyl)amine, the nanocrystals could also be transferred in water. However in this case a drastic loss in the fluorescence quantum efficiency from the $\mathrm{CdSe} / \mathrm{ZnS}$ nanocrystals was observed after the particles were run through the size exclusion column. A more systematic study of the cross-linker structural parameters influencing the nanocrystal solubility and their fluorescence efficiency (for semiconductors) is in progress.

\section{Polymer coating of CdSe/ZnS nanocrystals}

The concentration of the $\mathrm{CdSe} / \mathrm{ZnS}$ nanocrystal $(7.0 \mathrm{~nm})$ solutions was determined by absorption measurements. In the case of nanocrystals with an average diameter of $7.0 \mathrm{~nm}$ (first exciton peak at $641 \mathrm{~nm}$ in the absorption spectrum) an extinction coefficient of $1030000 \mathrm{M}^{-1} \mathrm{~cm}^{-1}$ was assumed, as reported in the literature ${ }^{6}$. In order to precipitate the particles and to remove any excess surfactant, $10 \mathrm{ml}$ of methanol were added to $600 \mu \mathrm{l}$ of CdSe/ZnS nanocrystal solution (20 $\mu \mathrm{M}$ in chloroform). After centrifugation the supernatant was discarded. $2.24 \mathrm{ml}$ of polymer solution and $3.76 \mathrm{ml}$ of chloroform were added to the precipitate, resulting in a total volume of 6 $\mathrm{ml}$. The concentration of nanocrystals in this solution was $2 \mu \mathrm{M}$, which was sufficiently low to avoid cross-linking effects among nanocrystals. After stirring for two hours, the solvent was slowly evaporated. $1.52 \mathrm{ml}$ of cross-linker solution was added to the resulting powder and the solution was sonicated for 20 minutes. The solvent was again removed by evaporation, $5 \mathrm{ml}$ of buffer solution were added to the solid and the sample was sonicated for 5 minutes. The solution was further diluted to $10 \mathrm{ml}$ by the addition of water and filtered through a syringe filter. Before filtration the sample was cloudy, but after filtration it became optically clear. Ten additional ml of water were added and the solution was placed in a centricon centrifuge filter tube. The sample was then centrifuged until the volume was reduced to $5 \mathrm{ml}$. Additional water was added and the centrifuge step was repeated. When the volume of the sample was of the order of 2-4 ml, the sample was run twice on a size exclusion column. The final concentration of the sample could be finely adjusted by further centrifugation in a centricon tube. 


\section{Polymer coating of Au nanocrystals}

The concentration of colloidal gold nanocrystal solution (4.0 $\mathrm{nm}$ average diameter) was determined by UV-visible absorption measurements, assuming that gold particles with a diameter of $4 \mathrm{~nm}$ have an extinction coefficient of $8.7 \bullet 10^{6} \mathrm{M}^{-1} \mathrm{~cm}^{-1}$ at their plasmon peak. $5 \mathrm{ml}$ of cold methanol were added to $250 \mu \mathrm{l}$ of gold solution $(11 \mu \mathrm{M})$, and the solution was centrifuged in order to precipitate the nanocrystals. The supernatant was discarded. $210 \mu \mathrm{l}$ of polymer solution were added to the precipitate and the solution was diluted to $5 \mathrm{ml}$ volume by the addition of chloroform. The concentration of gold nanocrystals in this solution was $0.55 \mu \mathrm{M}$, which was sufficiently low to avoid cross-linking effects among the particles. After stirring for two hours, the solvent was slowly evaporated. $140 \mu \mathrm{l}$ of cross-linker solution were added to the solid and the solution was sonicated for 20 minutes. The solvent was again removed by evaporation, $5 \mathrm{ml}$ of buffer solution were added to the solid, and the solution was sonicated for 5 minutes. It was then further diluted to $10 \mathrm{ml}$ by the addition of water and filtered through a syringe filter. After filtration, 10 additional $\mathrm{ml}$ of water were added and the solution was placed in a centricon tube. The sample was then centrifuged until the volume was reduced to $5 \mathrm{ml}$. Additional water was added and the centrifugation step was repeated. When the volume of the sample was of the order of $2 \mathrm{ml}$, it was removed from the tube and run twice on a size exclusion column. The final concentration of the sample could be finely adjusted by an additional centrifugation in a centricon centrifuge filter tube.

\section{Polymer coating of $\mathrm{CoPt}_{3}$ nanocrystals}

The concentration of the $\mathrm{CoPt}_{3}$ nanocrystal solution was determined by gravimetric measurements. The number of nanoparticles in the solution was in fact estimated by evaluating the average weight of a single nanocrystal and the total amount of nanocrystals in the solution. The density of $\mathrm{CoPt}_{3}$ is $18.86 \mathrm{~g} / \mathrm{cm}^{3}$ (as reported in the literature), so that the mass of a single $\mathrm{CoPt}_{3}$ nanocrystals having a diameter of $8.0 \mathrm{~nm}$ was estimated to be $5.43 \times 10^{-21} \mathrm{~kg}$. This calculation does not take into account the surfactant coating, whose contribution however is considered to be negligible.

Ten $\mathrm{ml}$ of isopropanol were added to $3 \mathrm{ml}$ of cobalt platinum solution $(2 \mu \mathrm{M})$, and the solution was centrifuged in order to precipitate the nanocrystals. The supernatant was discarded. $1.44 \mathrm{ml}$ of polymer solution was added to the precipitate and the volume was adjusted to $6 \mathrm{ml}$ by the addition of chloroform. The concentration of $\mathrm{CoPt}_{3}$ in this solution was $1 \mu \mathrm{M}$ and this was sufficiently low to avoid cross-linking effects among the particles. After stirring for two hours, the solvent was slowly evaporated under reduced pressure. To the resulting solid $0.98 \mathrm{ml}$ of cross-linker solution was added and the sample was sonicated for 20 minutes. Shortly after, the solvent was again removed and $5 \mathrm{ml}$ buffer solution were added to the solid. The sample was then sonicated for $5 \mathrm{~min}$, it was further diluted with water to $10 \mathrm{ml}$ and filtered through syringe filters. Ten additional $\mathrm{ml}$ of water were added and the solution was placed in a centricon tube. The sample was then centrifuged until the volume was reduced to $5 \mathrm{ml}$. Additional water was added and the centrifuge step was repeated. When the volume of the sample was of the order of 2-5 $\mathrm{ml}$, the sample was run twice on a size exclusion column. The concentration of the sample could be adjusted by removing some of the solvent using centricon centrifuge tubes. 


\section{Polymer coating of $\mathrm{Fe}_{2} \mathrm{O}_{3}$ nanocrystals}

The concentration of the $\gamma-\mathrm{Fe}_{2} \mathrm{O}_{3}$ nanocrystal solution $(9.2 \mathrm{~nm}$ average diameter) was also determined by gravimetric measurements. The density of the $\gamma-\mathrm{Fe}_{2} \mathrm{O}_{3}$ (maghemite) is $5 \mathrm{~g} / \mathrm{cm}^{3}$, so the mass of a single $\gamma-\mathrm{Fe}_{2} \mathrm{O}_{3}$ nanocrystal was calculated to be $1.91 \times 10^{-21} \mathrm{~kg}$. Once again, this calculation did not take into account the surfactant coating.

Six ml of methanol were added to $845 \mu$ of $\gamma-\mathrm{Fe}_{2} \mathrm{O}_{3}$ solution $(7.1 \mu \mathrm{M})$, and the solution was centrifuged in order to precipitate the nanocrystals. The supernatant was discarded. $1.68 \mathrm{ml}$ of polymer solution $(400 \mathrm{mg} / 10 \mathrm{ml})$ was added to the precipitate and the volume was adjusted to 6 $\mathrm{ml}$ by the addition of chloroform. The concentration of $\gamma-\mathrm{Fe}_{2} \mathrm{O}_{3}$ nanocrystals in this solution was $1 \mu \mathrm{M}$, sufficiently low to avoid cross-linking effects among the particles. After stirring for two hours the solvent was slowly evaporated under reduced pressure. $1.14 \mathrm{ml}$ of cross-linker solution $(43 \mathrm{mg} / 10 \mathrm{ml})$ was added to the solid and the resulting solution was sonicated for 20 minutes. Shortly after, the solvent was again removed by evaporation and $5 \mathrm{ml}$ of buffer solution were added to the solid. The sample was sonicated for $5 \mathrm{~min}$, it was further diluted with water to $10 \mathrm{ml}$ and then filtered through syringe filters. Ten additional $\mathrm{ml}$ of water were added and the solution was placed in a centricon tube. The sample was then centrifuged until the volume was reduced to $5 \mathrm{ml}$. Additional water was added and the centrifugation step was repeated. When the volume of the sample was of the order of 2-5 $\mathrm{ml}$, the sample was run twice on a size exclusion column. The concentration of the sample could be adjusted by removing some of the solvent using a centricon centrifuge filter tube. 


\section{Transmission electron microscopy (TEM)}

Transmission electron microscope images were taken on a Philips CM 100 electron microscope operated at an accelerating voltage of $100 \mathrm{KV}$. The nanocrystals were deposited from a dilute solution onto a 3-4 nm thick film of amorphous carbon supported on a 400 mesh copper grid (Ted Pella Inc., \# 01822-F). One drop of nanocrystal solution was deposited onto the grid and the solvent was evaporated. In the case of nanocrystals in water the grids were kept under ethanol vapors for 1-2 days prior to sample deposition. This treatment made the carbon film more hydrophilic and improved the adsorption of the nanocrystals on it. The microscope magnification was calibrated with a calibration grid (Grating Replica, Waffle, $21601 / \mathrm{mm}$, on 3mm grid, Ted Pella Inc., \# 607). The average sizes and size distributions of the various nanocrystal samples were estimated by analyzing the scanned TEM negatives with "ImageJ" image analysis software, freely available from $h t t p: / / r s b . i n f o . n i h . g o v / i j /$.

\section{Gel electrophoresis}

Gel electrophoresis was used to study the size and surface charge distribution of the polymer coated nanocrystals and to check for the presence of nanocrystal aggregates. The nanocrystals were diluted in a loading buffer containing glycerol (final glycerol concentration 5-10\%) and were run in a $0.5 \times$ TBE buffer on a $2 \%$ agarose gel, at $100 \mathrm{~V}$ for 1 hour. In the case of $\mathrm{CdSe} / \mathrm{ZnS}$ nanocrystals, the gel was illuminated with an ultraviolet trans-illuminator to image the

fluorescence from the bands. In the case of $\mathrm{CoPt}_{3}, \mathrm{Fe}_{2} \mathrm{O}_{3}$ and $\mathrm{Au}$ nanocrystals, the gels were imaged with a visible-light illuminator. 


\section{Fluorescence Correlation Spectroscopy: theory and experiments}

FCS was established by Madge et al. (1972) ${ }^{12}$ as a way for measuring the diffusion constants of fluorescent particles dispersed in a solvent. From the diffusion constant of a particle it is possible to derive then its hydrodynamic radius through the Stokes-Einstein relation. Here, we first give a short overview of the FCS theory (details are discussed elsewhere ${ }^{13-15}$ ), and then we describe the experimental procedure for determining the average radius of the nanocrystals in the present work, using the FCS theory.

Let us assume that an excitation light source is focused on a tiny volume $\left(\mathrm{V}_{\text {eff }}\right)$ of the solution containing the fluorescent particles. On average, $<\mathrm{N}>$ particles will be sampled in this volume. However, the number of particles $\mathrm{N}(\mathrm{t})$ in this volume will fluctuate over time, because the particles can diffuse in and out of it. In the case of dilute solutions (so only few particles are present in the focus volume, on average), the fluctuation in the number of particles can be described by a Poisson distribution:

$$
\sqrt{\frac{\left\langle(\delta \mathrm{N})^{2}\right\rangle}{\mathrm{N}}}=\frac{1}{\sqrt{\mathrm{N}}}
$$

$\delta \mathrm{N}(\mathrm{t})=\mathrm{N}(\mathrm{t})-<\mathrm{N}\rangle$ is the fluctuation of the number of particles, and $\langle\mathrm{N}\rangle$ is the mean number of particles in the volume. Now, the concentration of the particles must be high enough to guarantee a good signal to noise ratio, but low enough to observe free particle diffusion. A good compromise would correspond roughly to 1 particle per femtoliter of the focus-volume $V_{\text {eff. }}$ The FCS theory demonstrates that it is possible to derive the diffusion constant of the particles from $\delta \mathrm{N}(\mathrm{t})$, and the principle can be understood in terms of a simple model. The time a particle needs to diffuse in and out of the focus volume depends indeed on its diffusion coefficient. The larger the diffusion coefficient, the faster the particles can diffuse in and out of the focus, and consequently faster time scale will characterize the fluctuations of the number of particles in $V_{\text {eff. }}$ The analysis of the time scales involved in the fluctuations can be carried out by means of an autocorrelation function.

To a first approximation, we assume a constant value for the fluorescence emission from each particle inside the focus volume. In this simplified approach fluorescence fluctuations due to fluctuations in the absorption cross-section and in the quantum yield and blinking of the particles are neglected. Particles are also assumed to have a spherical shape.

The number of particles in the focus at a given time $t$ can be experimentally determined by the total fluorescence $F(t)$ collected. Since the excitation light is focused on a tiny volume $V_{\text {eff, }}$ only the particles within this volume contribute to the fluorescence signal. The fluctuation $\delta \mathrm{F}(\mathrm{t})=\mathrm{F}(\mathrm{t})$ $-\langle\mathrm{F}\rangle$ of the fluorescence signal is defined as the deviation from the mean fluorescence signal $\langle\mathrm{F}\rangle$, which is given by:

$$
\langle\mathrm{F}\rangle=\frac{1}{\mathrm{~T}} \int_{0}^{\mathrm{T}} \mathrm{F}(\mathrm{t}) \mathrm{dt}
$$


The number of particles $\mathrm{N}(\mathrm{t})$ in the focus can be written as an integral over the local particle concentration $\mathrm{c}(\underline{\mathrm{r}}, \mathrm{t})$ :

$$
\mathrm{N}(\mathrm{t})=\int_{\mathrm{V}_{\mathrm{eff}}} \mathrm{c}(\underline{\mathrm{r}}, \mathrm{t}) \mathrm{dV}
$$

We now assume that the fluctuations in the fluorescence signal are only due to local changes in the concentration $\delta c(\underline{r}, t)$ in the effective focus-volume $V_{\text {eff }}$. In addition, we merge the spatial parameters of the illumination to a function $\mathrm{W}(\underline{\mathrm{r}})$ that is written as:

$$
\mathrm{W}(\underline{\mathrm{r}})=\mathrm{e}^{-2\left(\mathrm{x}^{2}+\mathrm{y}^{2}\right) / \mathrm{r}_{0}^{2}} \cdot \mathrm{e}^{-2 \mathrm{z}^{2} / \mathrm{z}_{0}^{2}}
$$

W(r) is the so called Molecule Detection Efficiency which gives the probability of exciting and detecting a fluorescent particle in the solution. It is therefore the product of the excitation profile of the focused laser and the spatial collection efficiency of the confocal detection optics. Under carefully chosen setup conditions $\mathrm{W}(\underline{\mathrm{r}})$ can be approximated as a 3-dimensional Gaussian ellipsoid, as has been assumed in Equation (4). $\underline{r}=(x, y, z)$ describes the coordinates in the threedimensional space. Here $r_{0}$ is the radius in the focal plane where the excitation intensity of the laser has dropped to $1 / \mathrm{e}^{2}$ compared to the center. The parameter $\mathrm{z}_{0}$ gives the extension of the effective detection volume on the z-axis and is mainly determined by the used objective and the size of the pinhole.

The fluctuation of the fluorescence signal can then be written as:

$$
\delta \mathrm{F}(\mathrm{t})=\eta \int_{\mathrm{V}_{\mathrm{eff}}} \mathrm{W}(\underline{\mathrm{r}}) \cdot \delta \mathrm{c}(\underline{\mathrm{r}}, \mathrm{t}) \mathrm{dV}
$$

The constant value $\eta$ contains the quantum efficiency of the dye, detection efficiency and absorption cross-section. The normalized autocorrelation function $G(\tau)$ for fluorescence fluctuations $\delta \mathrm{F}(\mathrm{t})$ is defined as:

$$
\mathrm{G}(\tau)=\frac{\langle\delta \mathrm{F}(\mathrm{t}) \cdot \delta \mathrm{F}(\mathrm{t}+\tau)\rangle}{\langle\mathrm{F}(\mathrm{t})\rangle^{2}}
$$

$\mathrm{G}(\tau)$ is a measure of the self-similarity of the fluorescence signal after a delay-time $\tau$. Thus $\mathrm{G}(0)$ is the variance $\left.<\delta \mathrm{F}(\mathrm{t})^{2}>/<\mathrm{F}\right\rangle^{2}$. By inserting equation (5) in equation (6) the autocorrelation function becomes:

$$
\mathrm{G}(\tau)=\frac{\iint_{\mathrm{V} \mathrm{V}^{\prime}} \mathrm{W}(\underline{\mathrm{r}}) \cdot \mathrm{W}\left(\underline{\mathrm{r}}^{\prime}\right) \cdot\left\langle\delta \mathrm{c}(\underline{\mathrm{r}}, 0) \cdot \delta \mathrm{c}\left(\underline{\mathrm{r}}^{\prime}, \tau\right)\right\rangle \mathrm{dVdV^{ \prime }}}{\left(\langle\mathrm{c}\rangle \int_{\mathrm{V}} \mathrm{W}(\underline{\mathrm{r}}) \mathrm{dV}\right)^{2}}
$$


Under the assumption that the particles can diffuse freely in all three spatial directions (Brownian diffusion), we can derive an expression for the concentration fluctuations $\delta c(\underline{r}, t)$ if we solve the diffusion equation, where $\mathrm{D}$ is the diffusion coefficient.

$\frac{\delta \mathrm{c}(\underline{\mathrm{r}}, \mathrm{t})}{\delta \mathrm{t}}=\mathrm{D} \nabla^{2} \delta \mathrm{c}(\underline{\mathrm{r}}, \mathrm{t})$

We can use the following expression for $\delta \mathrm{c}(\underline{\mathrm{r}}, \mathrm{t})$ :

$\delta \mathrm{c}(\underline{\mathrm{r}}, \mathrm{t})=\delta \mathrm{c}(\underline{\mathrm{r}}, 0) \cdot \mathrm{e}^{-\underline{r}^{2} / \mathrm{Dt}}$

The solution of the diffusion equation using (9) yields:

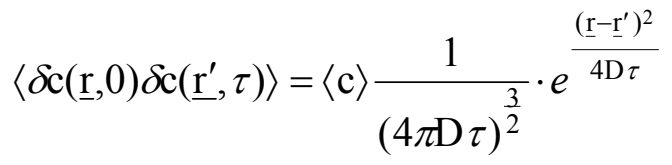

Insertion of (10) into (7), followed by integration over the volume, finally leads to the autocorrelation function for freely diffusing particles (11).

$$
\mathrm{G}(\tau)=\mathrm{G}_{\text {motion }}(\tau)=\frac{1}{\mathrm{~V}_{\text {eff }}\langle\mathrm{c}\rangle} \cdot \frac{1}{\left(1+\frac{\tau}{\tau_{\mathrm{D}}}\right)} \cdot \frac{1}{\sqrt{1+\frac{\tau}{\tau_{\mathrm{D}} \cdot \mathrm{s}^{2}}}}
$$

with the characteristic diffusion-time $\tau_{\mathrm{D}}$ given by

$$
\tau_{\mathrm{D}}=\frac{\mathrm{r}_{0}^{2}}{4 \mathrm{D}}
$$

and the structure parameter s given by

$$
\mathrm{s}=\frac{\mathrm{z}_{0}}{\mathrm{r}_{0}}
$$

The effective focus volume $\mathrm{V}_{\text {eff }}$ is then defined as

$$
\mathrm{V}_{\mathrm{eff}}=\frac{\left(\int_{V} \mathrm{~W}(\underline{\mathrm{r}}) \mathrm{dV}\right)^{2}}{\int_{V} \mathrm{~W}^{2}(\underline{\mathrm{r}}) \mathrm{dV}}=\frac{\left(\int \mathrm{e}^{-2^{\frac{\mathrm{x}^{2}+\mathrm{y}^{2}}{\mathrm{r}_{0}^{2}}}} \cdot \mathrm{e}^{-2 \frac{\mathrm{z}^{2}}{\mathrm{z}_{0}^{2}}} \mathrm{dV}\right)^{2}}{\int \mathrm{e}^{-4^{\frac{\mathrm{x}^{2}+\mathrm{y}^{2}}{\mathrm{r}_{0}^{2}}}} \cdot \mathrm{e}^{-4 \frac{\mathrm{z}^{2}}{\mathrm{z}_{0}^{2}}} \mathrm{dV}}=\pi^{\frac{3}{2}} \cdot \mathrm{r}_{0}^{2} \cdot \mathrm{z}_{0}
$$


In a more advanced approach, the correlation function $G_{\text {motion }}$ (which describes only changes in fluorescence due to the motion of the particles) is multiplied by a kinetic factor $X(\tau)$

$X(\tau)=\frac{1-\mathrm{T}_{\text {trip }}+\mathrm{T}_{\text {trip }} \mathrm{e}^{\tau / \tau_{\text {trip }}}}{1-\mathrm{T}_{\text {trip }}}$

which accounts for blinking / flickering ${ }^{15,16}$ in the fluorescence of the particles. The corrected autocorrelation function becomes then:

$\mathrm{G}_{\text {total }}(\tau)=\mathrm{G}_{\text {motion }}(\tau) \cdot \mathrm{X}(\tau)$

The hydrodynamic radius $r_{h}$ of the spherical particles can be obtained by inserting the diffusion constant D (from equation (12)) into the Stokes-Einstein-equation:

$\mathrm{r}_{\mathrm{h}}=\frac{\mathrm{kT}}{6 \pi \eta \mathrm{D}}$

Having given a brief overview of the FCS theory, we now describe the experimental procedure followed for the determination of the average diameter of the polymer coated nanocrystals synthesized in the present work.

Figure 1 shows a sketch of the experimental setup. A laser beam is focused by an objective with a high numerical aperture. Fluorescent light that is collected by the same objective can pass through the dichroic mirror as it is slightly shifted to higher wavelengths compared to the excitation. A 50/50 beamsplitter gives half of the intensity to two independent detection channels. With the filters the desired detection wavelengths can be chosen. Behind the pinholes of variable size the photons are detected with avalanche photodiodes giving the intensity versus time $F(t)$. An autocorrelation of the signal is done by a correlator card attached to a computer.

The Experiments were performed with a Confocor2 FCS-setup from Zeiss ${ }^{\mathrm{TM}}$. The laser light from an Ar-ion-Laser (Lasos/Zeiss) was coupled into the system via a dichroic mirror and was focused with a Zeiss C-Apochromat water immersion objective (40x; numerical aperture: 1.2) to a small volume within the diluted sample. The fluorescence signal emitted from the particles passed through a dichroic mirror and the focal plane was selected by a pinhole with $70 \mu \mathrm{m}$ diameter. The signal was split by a 50/50 beamsplitter and was collected by two Avalanche Photo Diodes (after passing appropriate filters). 


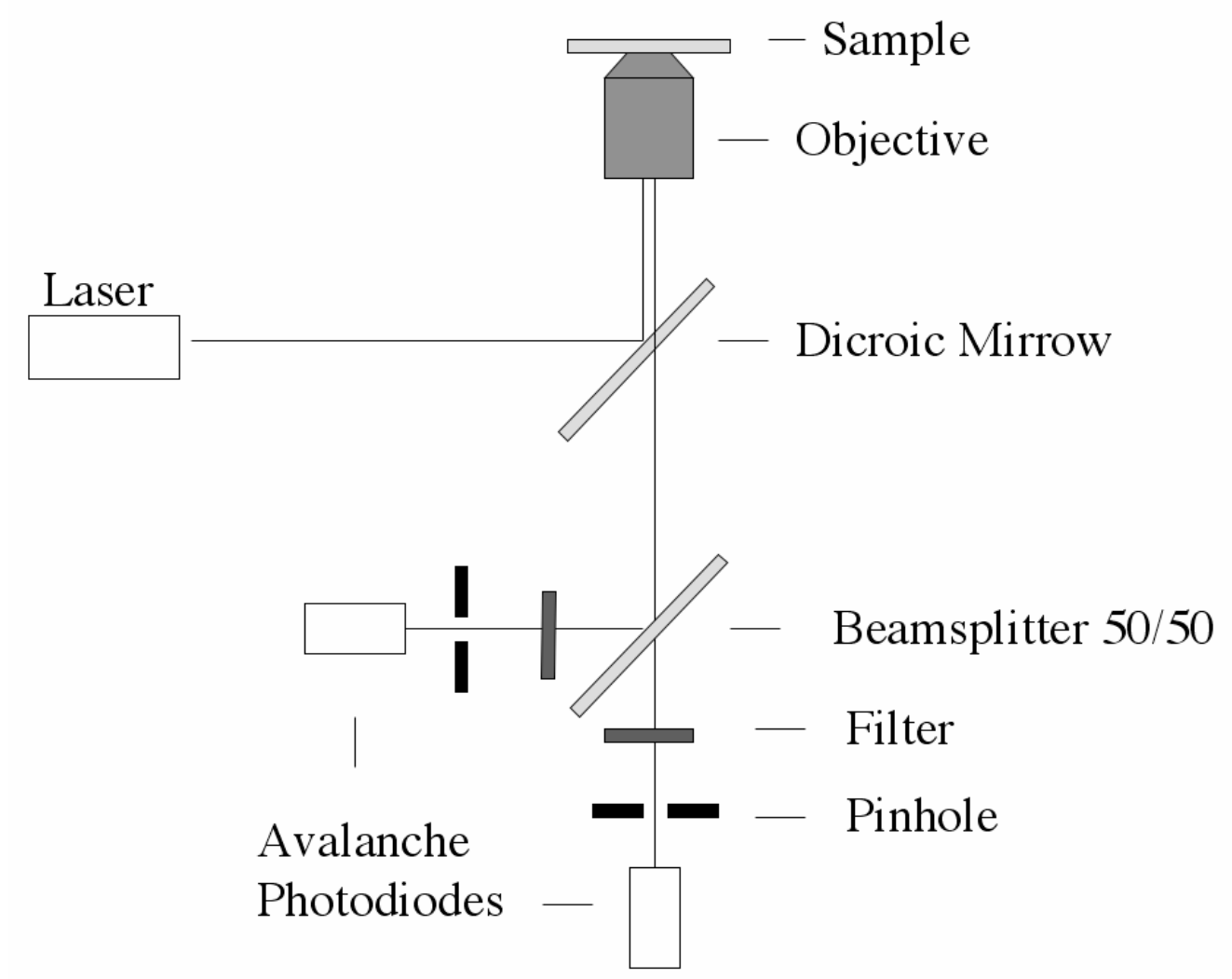

Figure 1. FCS setup. An Ar-ion-Laser (Lasos/Zeiss) was used, the cut-off wavelength of the dicroic mirror was 510 $\mathrm{nm}$, and $510 \mathrm{~nm}$ longpass filters were used to observe the emitted fluorescence.

First of all, the system had to be calibrated. In particular the volume of the focus had to be determined experimentally. For this purpose fluorescent molecules with a known diffusion constant were used as calibration sample. We used a solution of Alexa488 dye (Molecular Probes, excitation maximum $493 \mathrm{~nm}$, emission maximum $517 \mathrm{~nm})$ dissolved in water $(10 \mathrm{nM}$ concentration). The time trace of the fluorescence signal $\mathrm{F}(\mathrm{t})$ from this sample was recorded 10 times, each time for 20 seconds (Figure 2). For each measurement, the autocorrelation function $\mathrm{G}(\tau)$ for the fluorescence fluctuations $\delta \mathrm{F}(\mathrm{t})$ was calculated by using equation (6) (Figure 3 ). The experimental data for the autocorrelation function were fitted with equations $(11,16)$, using the 5

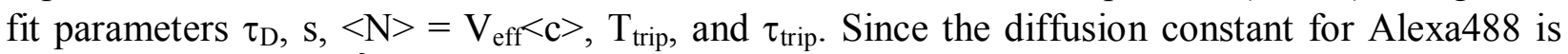
known $\left(D=316 \mu \mathrm{m}^{2} / \mathrm{s}\right)$, the focal radius $r_{0}$ could be derived via (12) using for $\tau_{D}$ the value obtained from the fit. Similarly, $z_{0}$ could be derived via (13) by using the fit results for the structure parameter $\mathrm{s}$ and $\mathrm{r}_{0}$. The mean values for $\mathrm{r}_{0}$ and $\mathrm{z}_{0}$ (and thus also for $\mathrm{s}$ ) obtained from the ten individual measurements where then used for all the measurements on nanocrystal samples. However, when other solvents than water were used, as in the case of nanocrystals dissolved in chloroform, the focal radius $r_{0}$ had to be adjusted for changes in the refractive index $n$ of the solvent:

$\mathrm{r}_{0}\left(\right.$ in $\left.\mathrm{H}_{2} \mathrm{O}\right) \cdot \mathrm{n}\left(\mathrm{H}_{2} \mathrm{O}\right)=\mathrm{r}_{0}($ in chloroform $) \cdot \mathrm{n}($ chloroform $)$

$\left(\mathrm{n}\left(\mathrm{H}_{2} \mathrm{O}\right)=1.33, \mathrm{n}(\right.$ chloroform $\left.)=1.45\right)$ 


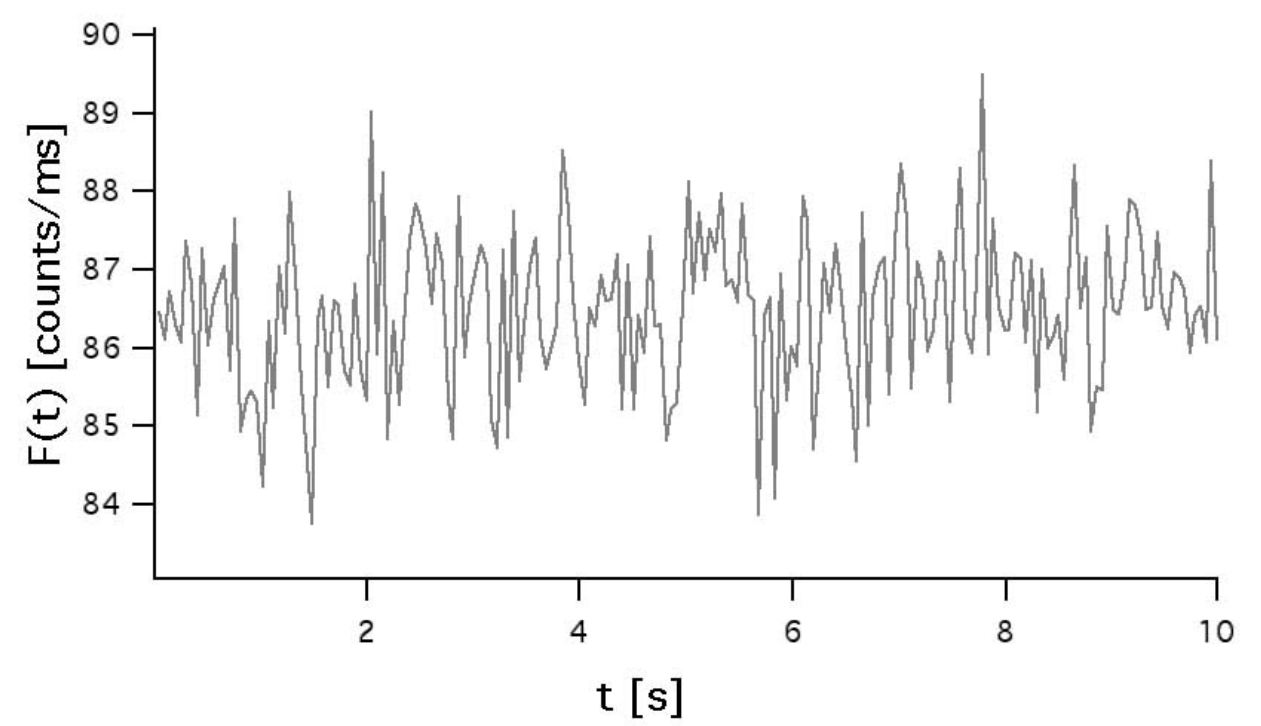

Figure 2: Time trace of the recorded fluorescence intensity $\mathrm{F}(\mathrm{t})$ of Alexa488 fluorescent dye.

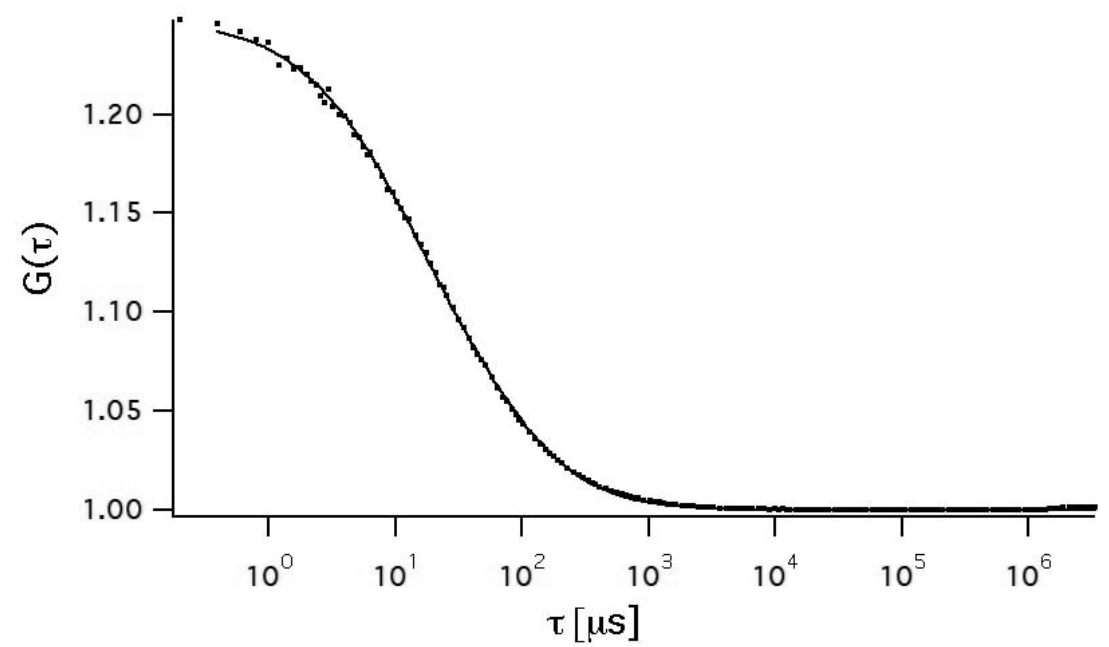

Figure 3: Autocorrelation function $\mathrm{G}(\tau)$ calculated from the $\mathrm{F}(\mathrm{t})$ data shown in Figure 2 (dotted line). The curve was fitted with the function given in equations $(11,16)$. The fit yielded the five fit parameters: characteristic diffusion time $\tau_{\mathrm{D}}=26.6 \mu \mathrm{s}$, structure parameter $\mathrm{s}=8.96$, average number of dye molecules within the focal volume $<\mathrm{N}>=$ $\mathrm{V}_{\text {eff }}<\mathrm{c}>=4.7, \mathrm{~T}_{\text {trip }}=0.15$, and $\tau_{\text {trip }}=4.7 \mu \mathrm{s}$. The focal radius $\mathrm{r}_{0}$ was calculated by means of (12), using the known diffusion-constant of Alexa488 ( $\mathrm{D}=316 \mu \mathrm{m}^{2} / \mathrm{s}$ ), and was equal to $183 \mathrm{~nm}$.

For the measurements on the nanocrystal samples, nanocrystal solutions with a concentration of approximately $50 \mathrm{nM}$ were deposited on a coverslip over the objective (the same as for the Alexa488 dye solution). The time trace of the fluorescence signal $\mathrm{F}(\mathrm{t})$ was recorded at least ten times, each time for 20 seconds. The corresponding autocorrelation functions $G(\tau)$ were calculated using equation (6) and were fitted with (11) and (16). This time only four fit parameters were used: $\tau_{\mathrm{D}},\langle\mathrm{N}\rangle=\mathrm{V}_{\text {eff }}<\mathrm{c}>, \mathrm{T}_{\text {trip }}$, and $\tau_{\text {trip }}$. The geometrical parameters for the focus volume ( $\mathrm{r}_{0}$ and $\mathrm{z}_{0}$, as well as $\mathrm{s}$ ) where in fact known from the previous calibration with the Alexa488 solution. The diffusion constant for the nanocrystals D could be calculated via (12), using the diffusion time $\tau_{\mathrm{D}}$, as determined from the fit, and the known focal radius $\mathrm{r}_{0}$. Finally, the hydrodynamic radius of the nanocrystals was calculated using the Stokes-Einstein-equation (17). For the samples dissolved in chloroform and for those dissolved in water the viscosities $\eta_{\mathrm{CHCl3}}=$ 
$0.57 \mathrm{mPa} \cdot \mathrm{s}$ and $\eta_{\text {water }}=0.98 \mathrm{mPa} \cdot \mathrm{s}$ respectively, were assumed at $20^{\circ} \mathrm{C}$. At least 10 individual measurements were performed for each sample, and the resulting hydrodynamic radius was determined as the mean value of the measurements.

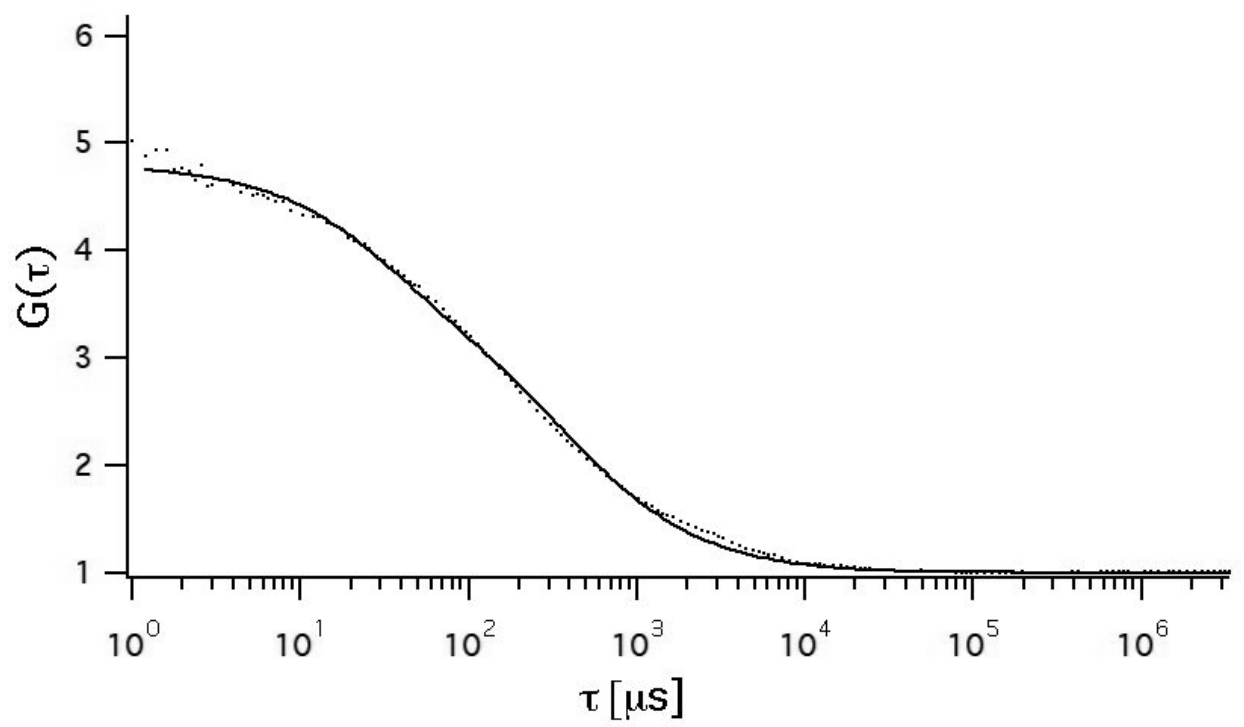

Figure 4: Autocorrelation function $\mathrm{G}(\tau)$ calculated from the $\mathrm{F}(\mathrm{t})$ data recorded on a sample of green fluorescent, polymer-coated $\mathrm{CdSe} / \mathrm{ZnS}$ nanocrystals dissolved in water (dotted line). The curve was fitted with the function given in equation $(11,16)$, whereby the structure parameter (as determined from the calibration with Alexa488) was kept fixed at $s=7.6$. This curve was actually recorded on a different day than the curve shown in Figure 3. Therefore a new calibration was necessary, and this yielded a structure parameter that was slightly different from the one reported in Figure 3. The fit yielded the following four free parameters: characteristic diffusion time $\tau_{\mathrm{D}}=327.5 \mu \mathrm{s}$, average number of dye molecules within the focal volume $<\mathrm{N}>=\mathrm{V}_{\text {eff }}<\mathrm{c}>=0.35, \mathrm{~T}_{\text {trip }}=0.34$ and $\tau_{\text {trip }}=30.1 \mu \mathrm{s}$. With the known value for $r_{0}=167 \mathrm{~nm}$ the diffusion constant was determined via (12) to be $\mathrm{D}=21.3 \mu \mathrm{m}^{2} / \mathrm{s}$. 
Table 1: Summary of all polymer coating experiments

\begin{tabular}{|c|c|c|}
\hline sample & $\frac{\text { polymer units }}{n \mathrm{~nm}^{2} \text { nanoparticle }}$ & $\frac{\text { [polymer chains] }}{\text { [crosslinker] }}$ \\
\hline $\mathrm{Fe}_{2} \mathrm{O}_{3}$ & 93 & 13 \\
\hline $\mathrm{Fe}_{2} \mathrm{O}_{3}$ & 100 & 10 \\
\hline $\mathrm{Fe}_{2} \mathrm{O}_{3}$ & 182 & 13 \\
\hline $\mathrm{Fe}_{2} \mathrm{O}_{3}$ & 260 & 13 \\
\hline $\mathrm{Fe}_{2} \mathrm{O}_{3}$ & 519 & 10 \\
\hline $\mathrm{Au}$ & 100 & 10 \\
\hline $\mathrm{Au}$ & 92 & 10 \\
\hline $\mathrm{Au}$ & 147 & 7 \\
\hline $\mathrm{Au}$ & 295 & 7 \\
\hline $\mathrm{Au}$ & 310 & 8 \\
\hline $\mathrm{CdSe} / \mathrm{ZnS}$ & 10 & 6 \\
\hline $\mathrm{CdSe} / \mathrm{ZnS}$ & 15 & 6 \\
\hline $\mathrm{CdSe} / \mathrm{ZnS}$ & 20 & 6 \\
\hline $\mathrm{CdSe} / \mathrm{ZnS}$ & 60 & 6 \\
\hline $\mathrm{CdSe} / \mathrm{ZnS}$ & 100 & 10 \\
\hline $\mathrm{CoPt}$ & 100 & 10 \\
\hline
\end{tabular}




\section{References}

(1) Shevchenko, E. V.; Talapin, D. V.; Rogach, A. L.; Kornowski, A.; Haase, M.; Weller, H. JACS 2002, 124, 11480-11485.

(2) Fink, J.; Kiely, C. J.; Bethell, D.; Schiffrin, D. J. Chem. Mat. 1998, 10, 922-926.

(3) Dabbousi, B. O.; Rodriguez-Viejo, J.; Mikulec, F. V.; Heine, J. R.; Mattoussi, H.; Ober, R.; Jensen, K. F.; Bawendi, M. G. J. Phys. Chem. B 1997, 101, 9463-9475.

(4) Hyeon, T.; Lee, S. S.; Park, J.; Chung, Y.; Na, H. B. J. Am. Chem. Soc. 2001, 123, 1279812801.

(5) Reiss, P.; Bleuse, J.; Pron, A. Nanoletters 2002, 2, 781-784.

(6) Yu, W. W.; Qu, L.; Guo, W.; Peng, X. Chem. Mat. 2003, 15, 2854-2860.

(7) Leff, D. V.; O'Hara, P. C.; Heath, J. R.; Gelbart, W. M. The Journal of Physical Chemistry 1995, 99, 7036-7041.

(8) Kiely, C. J.; Fink, J.; Zheng, J. G.; Brust, M.; Bethell, D.; Schiffrin, D. J. Adv. Mater. 2000, 12, 640-643.

(9) Templeton, A. C.; Wuelfing, W. P.; Murray, R. W. Accounts Chem. Res. 2000, 33, 27-36.

(10) Shevchenko, E. V.; Talapin, D. V.; Schnablegger, H.; Kornowski, A.; Festin, Ö.;

Svedlindh, P.; Haase, M.; Weller, H. JACS 2003, 125, 9090-9101.

(11) Zanchet, D.; Micheel, C. M.; Parak, W. J.; Gerion, D.; Williams, S. C.; Alivisatos, A. P. J. Phys. Chem. B 2002, 106, 11758-11763.

(12) Magde, D.; Elson, E.; Webb, W. W. Phys. Rev. Lett 1972, 29, 705-708.

(13) Eigen, M.; Rigler, R. Proc. Natl. Acad. Sci. USA 1994, 91, 5740-5747.

(14) Schwille, P.; Bieschke, J.; Oehlenschläger, F. Biophysical Chemistry 1997, 66, 211-228.

(15) Krichevsky, O.; Bonnet, G. Rep. Prog. Phys. 2002, 65, 251-297.

(16) Amediek, A.; Haustein, E.; Scherfeld, D.; Schwille, P. Single Molecules 2002, 3, 201-210. 